\title{
Medical Image Registration Based on BSP and Quad-Tree Partitioning
}

\author{
A. Bardera, M. Feixas, I. Boada, J. Rigau, and M. Sbert \\ Institut d'Informàtica i Aplicacions, Universitat de Girona, Spain \\ \{anton.bardera, miquel.feixas, imma.boada, \\ jaume.rigau, mateu.sbert\}@udg.es
}

\begin{abstract}
This paper presents a study of image simplification techniques as a first stage to define a multiresolution registration framework. We propose here a new approach for image registration based on the partitioning of the source images in binary-space (BSP) and quad-tree structures. These partitioned images have been obtained with a maximum mutual information gain algorithm. Multimodal registration experiments with downsampled, BSP and quadtree partitioned images show an outstanding accuracy and robustness by using BSP images, since the grid effects are drastically reduced. The obtained results indicate that BSP partitioning can provide a suitable framework for multiresolution registration.
\end{abstract}

\section{Introduction}

Multimodal image registration plays an increasingly important role in medical imaging. Its objective is to find a transformation that maps two or more images, acquired using different imaging modalities, by optimizing a certain similarity measure. Among the different similarity measures that have been proposed, mutual information (MI) 2, 9] and normalized mutual information (NMI) 6] are the most commonly used since they produce satisfactory results in terms of accuracy, robustness and reliability. However, MI-based methods are very sensitive to implementation decisions, such as interpolation and optimization methods, and multiresolution strategies [4. The latter allow us to reduce the computational cost by means of a coarse-to-fine hierarchical representation of the images. Crucial to building these hierarchies is the selection of the image simplification strategy.

The main objective of this paper is to analyze the behavior of the registration process when the source images are simplified in BSP and quad-tree structures, obtained with a maximum MI gain algorithm [5. We will see that multimodal registration experiments based on BSP partitioned images show a remarkable accuracy and robustness, reducing substantially the grid effects compared with both regular downsampled and quad-tree images. Since experimental results demonstrate the good performance using these simplification strategies, we suggest they are an ideal strategy for defining a multiresolution framework. Such a framework can be used not only for registration purposes but also for image processing or transmission in telemedicine environments. 
This paper is organized as follows. In Section 2, we briefly describe image registration and partitioning techniques using MI maximization. In Section 3, a new image registration framework based on partitioned images is presented. In Section 4, multimodal registration experiments show the suitability of the presented approach. Finally, our conclusions are given in Section 5.

\section{Previous Work}

In this section we review the MI definition [1] and its application to image registration [2, 9, 4, 7] and partitioning [5].

Mutual Information. Given two discrete random variables, $X$ and $Y$, with values in the sets $\mathcal{X}=\left\{x_{1}, \ldots, x_{n}\right\}$ and $\mathcal{Y}=\left\{y_{1}, \ldots, y_{m}\right\}$, respectively, the MI between $X$ and $Y$ is defined as

$$
I(X, Y)=\sum_{i=1}^{n} \sum_{j=1}^{m} p_{i j} \log \frac{p_{i j}}{p_{i} q_{j}}
$$

where $n=|\mathcal{X}|, m=|\mathcal{Y}|, p_{i}=\operatorname{Pr}\left[X=x_{i}\right]$ and $q_{j}=\operatorname{Pr}\left[Y=y_{j}\right]$ are the marginal probabilities and $p_{i j}=\operatorname{Pr}\left[X=x_{i}, Y=y_{j}\right]$ is the joint probability. $I(X, Y)$ is a measure of the shared information between $X$ and $Y$. It can also be expressed as $I(X, Y)=H(X)-H(X \mid Y)=H(Y)-H(Y \mid X)$, where $H(X)$ and $H(Y)$ are the marginal entropies, and $H(X \mid Y)$ and $H(Y \mid X)$ the conditional entropies [1.

A fundamental property of $\mathrm{MI}$ is the data processing inequality which can be expressed in the following way: if $X \rightarrow Y \rightarrow Z$ is a Markov chain, then

$$
I(X, Y) \geq I(X, Z) .
$$

This result demonstrates that no processing of $Y$, deterministic or random, can increase the information that $Y$ contains about $X$ [1].

MI-based Image Registration. The most successful automatic image registration methods are based on MI, which is a measure of the dependence between two images. The registration of two images is represented by an information channel $X \rightarrow Y$, where the random variables $X$ and $Y$ represent the images. Their marginal probability distributions, $\left\{p_{i}\right\}$ and $\left\{q_{j}\right\}$, and the joint probability distribution, $\left\{p_{i j}\right\}$, are obtained by simple normalization of the marginal and joint intensity histograms of the overlapping areas of both images [2]. The registration method based on the maximization of MI, almost simultaneously introduced by Maes et al. 2] and Viola et al. 9, is based on the conjecture that the correct registration corresponds to the maximum $M I$ between the overlapping areas of the two images. Later, Studholme et al. [6] proposed a normalization of MI defined by

$$
N M I(X, Y)=\frac{H(X)+H(Y)}{H(X, Y)}=1+\frac{I(X, Y)}{H(X, Y)},
$$

which is more robust than MI, due to its greater independence of the overlap area. 
The behavior of the MI-based method depends on the implementation decisions. Thus, for instance, it is especially sensitive to the interpolator and optimizator chosen or the binning and multiresolution strategies [4. Generally the grid points of the transformed image do not coincide with the grid points of the reference image. Thus, the selection of an interpolator is required. Although there are different interpolators, all of them introduce artifacts due to the error patterns caused by the grid regularity [7. On the other hand, the simple computation of an MI-based similarity measure by sampling the images on a regular grid leads to undesired artifacts, called grid effects [8].

MI-Based Partitioning Algorithm. An MI-based algorithm was presented by Rigau et al. [5] to partition an image. Given an image with $N$ pixels and an intensity histogram with $n_{i}$ pixels in bin $i$, a discrete information channel $X \rightarrow Y$ is defined, where $X$ represents the bins of the histogram, with marginal probability distribution $\left\{p_{i}\right\}=\left\{\frac{n_{i}}{N}\right\}$, and $Y$ the image partitioned into pixels, with uniform distribution $\left\{q_{j}\right\}=\left\{\frac{1}{N}\right\}$. The conditional probability distribution $\left\{p_{j \mid i}\right\}$ of this channel is defined as the transition probability from bin $i$ of the histogram to pixel $j$ of the image, and vice versa for $\left\{p_{i \mid j}\right\}$. This channel fulfills that $I(X, Y)=H(X)$ since, knowing the output (pixel), there is no uncertainty about the input bin of the histogram. From the data processing inequality (2), any clustering or quantization over $X$ or $Y$, respectively represented by $\widehat{X}$ and $\widehat{Y}$, will reduce the MI of the channel. Thus, $I(X, Y) \geq I(X, \widehat{Y})$ and $I(X, Y) \geq$ $I(\widehat{X}, Y)$.

From the above reasonings, a pixel clustering algorithm which minimizes the loss of MI could be proposed. However, its high cost suggests adopting the contrary strategy, where the full image is taken as the unique initial partition and is progressively subdivided according to the maximum MI gain for each partitioning step. This algorithm is a greedy top-down procedure which partitions an image in quasi-homogeneous regions. This method can be visualized from equation $H(X)=I(X, \widehat{Y})+H(X \mid \widehat{Y})$, where the acquisition of information increases $I(X, \widehat{Y})$ and decreases $H(X \mid \widehat{Y})$, producing a reduction of uncertainty due to the equalization of the regions. Different stopping criteria can be used. For more details, see [5].

\section{Registration from Partitioned Images}

Registration aims to find a transformation which maps two or more images by optimizing certain similarity measure. Multiresolution and multisampling strategies can be used to reduce its computational cost by means of a coarse-to-fine hierarchical strategy which starts with the reference and floating images on a coarser resolution. The estimates of the correspondence or parameters of the mapping functions while going up to finer resolutions are progressively improved. At every level they considerably decrease the search space and thus save computational time. In particular, downsampling techniques cause a great acceleration of the registration process [4]. 




(a) $\mathrm{MR}$

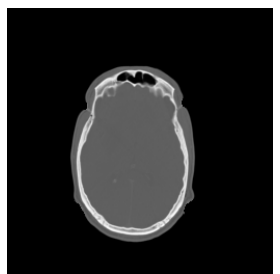

(b) $\mathrm{CT}$

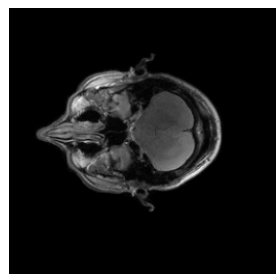

(c) MR

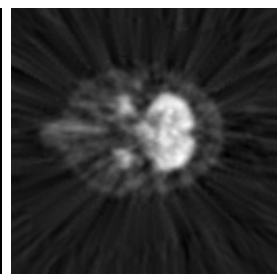

(d) PET

Fig. 1. Test images from the Vanderbilt database 3

Obviously, a good strategy to speed-up the registration process could be to use simplified images instead of the original ones. Our proposal is to register the images obtained with the MI-based partitioning algorithm presented in Sec. 2. These images contain a high information level for a reduced number of regions. This proposal is a first approximation for considering the benefits of a multiresolution approach which would consist in the interplay of the different resolutions of both images to accelerate registration. At each registration level, the best suited resolution for each image would be selected. Crucial to developing this multiresolution framework is the selection of the simplification strategy that has to be applied to simplify images. In this paper, we investigate two subdivision techniques, BSP and quadtree, to determine which provides better results.

To carry out this study, we propose a two step registration process. In the first step, the original images are progressively partitioned with vertical or horizontal

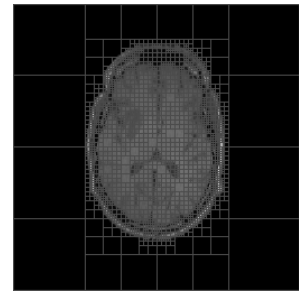

(i.a)

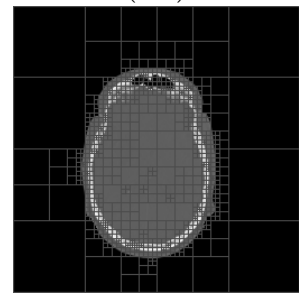

(ii.a)



$(i . b)$



(ii.b)

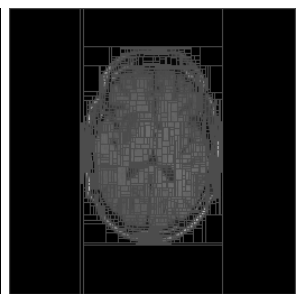

(i.c)

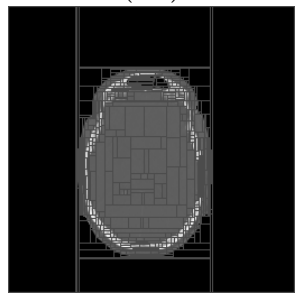

(ii.c)



(i.d)

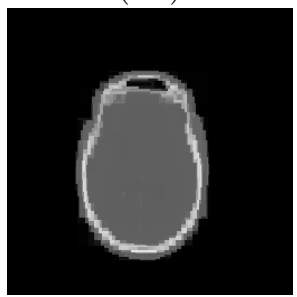

(ii.d)

Fig. 2. (i) MR and (ii) CT images obtained from Fig. 1 $(a-b)$. (a) Quad-tree partitions with $M I R=0.7,(b)$ quad-tree simplified images, $(c)$ BSP partitions with $M I R=0.7$, and $(d)$ BSP simplified images. 
Table 1. Percentage of regions obtained with respect to the initial number of pixels corresponding to MR and CT original images of Fig. 1 $(a-b)$ and for a given MIR

\begin{tabular}{|c||c|c||c|c|}
\hline \multicolumn{1}{|c||}{$M I R$} & \multicolumn{2}{c||}{ MR } & \multicolumn{2}{c|}{ CT } \\
\cline { 2 - 5 } & BSP & quad-tree & BSP & quad-tree \\
\hline \hline 0.5 & 0.25 & 0.40 & 0.06 & 0.13 \\
0.6 & 0.81 & 1.18 & 0.21 & 0.39 \\
0.7 & 2.21 & 3.16 & 0.77 & 1.28 \\
0.8 & 5.28 & 6.56 & 2.73 & 3.87 \\
0.9 & 11.88 & 16.05 & 7.98 & 11.48 \\
\hline
\end{tabular}

lines (BSP) or with a quad-tree structure. In both cases, an MI ratio given by $\operatorname{MIR}(X, \widehat{Y})=\frac{I(X, \widehat{Y})}{H(X)}$ is used as a stopping criterion. This ratio is a measure of the simplification quality.

In Fig. 2 we illustrate the behaviour of this partitioning step applying it to the 2D MR-CT pair of images (Fig. 1 $(a-b))$. In Fig. 2(a,c) we show for each original image the partitioning lines of the quad-tree and BSP structures and in Fig. 2(b,d) the corresponding simplified images obtained by averaging for each region the intensity of its pixels. We also collect in Table 1 the percentage of regions obtained with the simplification with respect to the initial number of pixels corresponding to the original MR and CT images. Note that a big gain of MI is obtained with a relative low number of partitions. Thus, for instance, in the $\mathrm{CT}$ case, a $70 \%$ of $\mathrm{MI}(M I R=0.7)$ is obtained with approximately $1 \%$ of the maximum number of partitions (number of pixels of the source image). Observe that less partitions are needed in the CT image to extract the same $M I R$ than in the MR image. This is due to the fact that the higher the image homogeneity, the higher the degree of simplification. In this example, the CT image is more homogeneous than the MR image.

In the second step of the process, the previously partitioned images are registered using the NMI metric and the Powell's algorithm as optimizer. To illustrate the feasibility of this proposal, we have registered simplified images of the MRCT of Fig. $1(a-b)$, considering first an MIR of 0.6 and then an MIR of 0.7. The registration results are shown in Fig. 3, where, respectively, $(a)$ and $(b)$ correspond to $M I R=0.6$ and $M I R=0.7$, and $(i)$ and $(i i)$ to the quad-tree and BSP partitioned images. In this figure, to illustrate better the obtained results, we apply the transformation obtained from the registration of the simplified images to the original ones. In addition, for each one of these images we compute the translational error $\left(t_{x}, t_{y}\right)$. We consider the registration result of the original images without any partitioning process as being correct, so this error measures the deviation in $x$ and $y$ translation between the transformation corresponding to the correct registration and the evaluated one. In all the cases, the rotational error has been omitted due to its insignificant value. Observe that BSP images with $M I R=0.6$ (Fig. $3($ ii.a $)$ ) achieve a lower error than quad-tree images with $M I R=0.7$ (Fig. $3(i . b)$ ). This demonstrates that better results are obtained with the registration of the BSP partitioned images. 


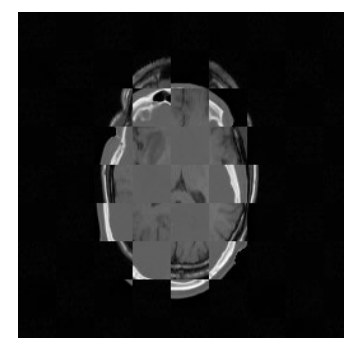

(i.a) $(5.64,16.33)$

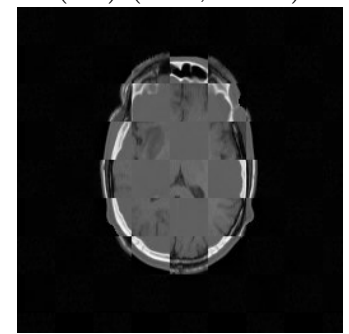

(ii.a) $(0.36,0.71)$



(i.b) $(2.38,0.36)$



(ii.b) $(0.36,0.17)$

Fig. 3. Registration for the MR-CT pair of Fig. 1 $(a-b)$. (i) Quad-tree and (ii) BSP subdivision methods for $(a) M I R=0.6$ and $(b) M I R=0.7$. The translational error $\left(t_{x}, t_{y}\right)$ is shown for each registration.

\section{Results and Discussion}

In order to evaluate more accurately the performance of the registration of MIbased partitioned images, experiments on MR-CT (Fig. 1( $(a-b)$ ) and MR-PET (Fig. 1 1 - d) images are presented. In these experiments, the corresponding pair of images have the same degree of simplification, i.e., an MR quad-tree (or BSP) image with $M I R=0.7$ is registered with a CT quad-tree (or BSP) with the same MIR. These results are compared with regular downsampled images.

In Fig. (4t the results of our experiments are presented. The behavior of the NMI measure is analyzed moving the floating image one pixel at each step through the $\mathrm{X}$ axis from -100 to 100 pixel units around the origin. No interpolation artifacts appear since there is no pixel interpolation. In all the plots, the bottom curve corresponds to the NMI registration of the source images. The MR-CT and MR-PET results are shown in the first $(i)$ and second (ii) rows, respectively. In Fig. $4(a)$, we illustrate the NMI measure obtained with different downsamplings of the original images. From bottom to top, the NMI curves correspond to downsampling of $2 \times 2,4 \times 4,8 \times 8$ and $16 \times 16$ pixels, respectively. Note that, high artifacts appear at every $n$ pixels coinciding with the downsampling factor. In Fig. 4(b-c), we illustrate the NMI values for the quad-tree and BSP partitioned images, respectively. Each curve corresponds to a different degree of simplification. From bottom to top, $M I R$ ranges from 0.9 to 0.5. Observe in Fig. 4( $b)$ that the quad-tree partition also produces correlation artifacts due to the regularity 


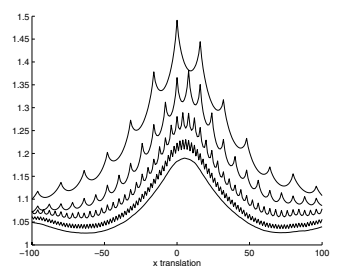

(i.a)

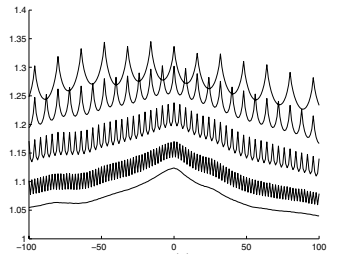

(ii.a)

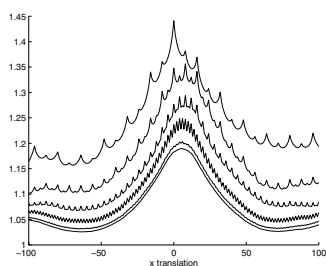

$(i . b)$

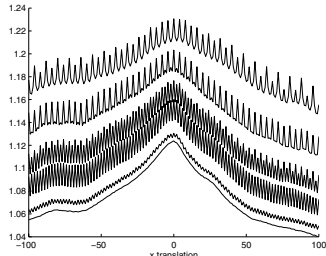

(ii.b)

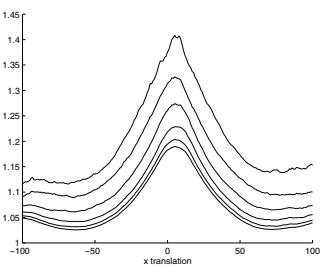

(i.c)

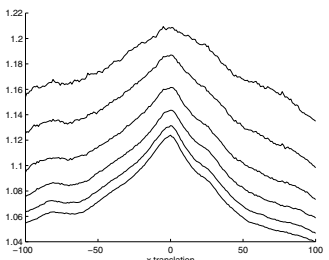

(ii.c)

Fig. 4. (i) MR-CT (Fig. 1 $(a-b))$ and (ii) MR-PET (Fig. 1 $(c-d)$ ) registration results corresponding to $(a)$ downsampled, $(b)$ quad-tree, and $(c)$ BSP images. The horizontal axis represents the slice translation on the X-axis (in pixels) and the vertical axis the value of NMI. For each plot, the NMI measure for different degrees of downsampling $(a)$ and simplification $(b-c)$ of the images are shown.

of its partitions. However, these artifacts are slightly reduced with respect to the downsampling case, since, although the registered images have the same degree of simplification, the number and the position of the generated quad-tree partitions are not the same.

Finally, in Fig. 4( $(c)$ we analyze the BSP partition. In this case, the grid artifacts are nearly completely eliminated since neither the position nor the number of partitions of the images coincide. Registration is more robust since the probability of finding a local maximum is lower as it is shown by the smoothness of BSP plots. Taking into account that the perfect registration is given by the maximum bottom curve, observe the high accuracy, i.e., the coincidence of the curve maxima, of the registration reached with the BSP images. For instance, an accurate registration is achieved with $M I R=0.7$, which represents an approximate reduction of $99 \%$ of the original number of pixels.

Experiments with the MR-PET images shown in Fig. 4( $i i)$ behave similarly to the MR-CT case in Fig. 4( $i$ ). In both cases, the BSP simplification scheme behaves considerably better than both quad-tree simplification and downsampled images in terms of the reduction of grid artifacts. From these experiments we can conclude that the BSP approach is more robust and accurate.

\section{Conclusions and Future Work}

In this paper, we have presented a new technique for image registration based on the partitioning of the source images. Thepartitioning algorithm relies on the 
maximization of the mutual information gain for each refinement decision. The presented method is a first step towards a full multiresolution registration approach. Two alternatives (binary space partition and quad-tree simplifications) have been analyzed and compared with a usual regular downsampling technique. The quality of the subdivision has been investigated in terms of the efficiency in registration. Results have shown the superior quality of the BSP subdivision, which allows a smoother registering. The BSP approach performs also better than regular downsampling. The next step in our research will consist in developing a multiresolution framework using the BSP subdivision.

\section{Acknowledgments}

The images used in our experiments were provided as part of the project, "Evaluation of Retrospective Image Registration", National Institutes of Health, Project Number 1 R01 NS33926-01, Principal Investigator Prof. J. Michael Fitzpatrick, Vanderbilt University, Nashville, TN. This project has been funded in part with grant numbers TIN2004-08065-C02-02, TIN2004-07451-C03-01 and 2001-SGR-00296.

\section{References}

1. Thomas M. Cover and Joy A. Thomas. Elements of Information Theory. Wiley Series in Telecommunications, 1991.

2. F. Maes, A. Collignon, D. Vandermeulen, G. Marchal, and P. Suetens. Multimodality image registration by maximization of mutual information. IEEE Transactions on Medical Imaging, 16(2):187-198, 1997.

3. National Institutes of Health. Retrospective Image Registration Evaluation. Vanderbilt University, Nashville (TN), USA, 2003. Project Number 8R01EB002124-03, Principal Investigator J. Michael Fitzpatrick.

4. Josien P.W. Pluim, J.B.A. Maintz, and M.A. Viergever. Mutual-information-based registration of medical images: a survey. IEEE Transactions on Medical Imaging, 22:986-1004, 2003.

5. J. Rigau, M. Feixas, M. Sbert, A. Bardera, and I. Boada. Medical image segmentation based on mutual information maximization. In International Conference on Medical Image Computing and Computed Assisted Intervention (MICCAI 2004), Proceedings, Rennes-Saint Malo, France, September 2004.

6. Colin Studholme. Measures of 3D Medical Image Alignment. PhD thesis, University of London, London, UK, August 1997.

7. J. Tsao. Interpolation artifacts in multimodal image registration based on maximization of mutual information. IEEE Transactions on Medical Imaging, 22:854-864, 2003.

8. M. Unser and P. Thévenaz. Stochastic sampling for computing the mutual information of two images. In Proceedings of the 5th International Workshop on Sampling Theory and Applications (SampTA'03), pages 102-109, Strobl, Austria, May 2003.

9. Paul A. Viola. Alignment by Maximization of Mutual Information. PhD thesis, Massachusetts Institute of Technology, Massachusetts (MA), USA, 1995. 